\title{
Quantifier les puits et sources des gaz à effet de serre : une nouvelle ambition pour la télédétection spatiale
}

\section{Frédéric Chevallier, Philippe Bousquet, François-Marie Bréon, Grégoire Broquet}

Laboratoire des sciences du climat et de l'environnement, Institut Pierre-Simon Laplace, CEA / CNRS / Université Versailles-Saint-Quentinen-Yvelines, Gif-sur-Yvette

frederic.chevallier@lsce.ipsl.fr

\section{Résumé}

La longue durée de vie de certains gaz à effet de serre dans l'atmosphère, comme le dioxyde de carbone, le méthane et le protoxyde d'azote, donne à ceux-ci un rôle critique dans l'évolution du climat. Par ailleurs, l'accumulation de ces gaz augmente le niveau de fond et réduit ainsi leur variabilité spatio-temporelle relative à celui-ci, ce qui conduit à des spécifications particulièrement ambitieuses sur la précision attendue des mesures, pour qu'elles apportent une information nouvelle. Leur télédétection spatiale a donc débuté tardivement, au cours de la décennie 2000. La mesure de leurs concentrations atmosphériques est essentiellement motivée par l'estimation de la quantité nette de gaz échangée entre l'atmosphère et la surface du globe. Un tel objectif impose la conception de chaînes de traitement sophistiquées, faisant appel à des modèles de chimie-transport atmosphérique et d'autres sources d'information complémentaires sur les flux de ces gaz.
$\mathrm{V}$ olontairement ou non, l'activité humaine rejette un grand nombre de substances dans l'atmosphère. Localement, ces rejets dégradent la qualité de l'air, provoquant par exemple l'augmentation des concentrations d'ozone, de dioxyde d'azote ou de particules. À l'échelle du globe, ils modifient le bilan radiatif du système Terre, majoritairement en augmentant les concentrations atmosphériques de gaz à effet de serre à longue durée de vie comme le dioxyde de carbone $\left(\mathrm{CO}_{2}\right)$, le méthane $\left(\mathrm{CH}_{4}\right)$ et le protoxyde d'azote $\left(\mathrm{N}_{2} \mathrm{O}\right)$. Ainsi, depuis le début de l'ère industrielle, les concentrations de $\mathrm{CO}_{2}$ ont augmenté de plus de $40 \%$, celles de $\mathrm{CH}_{4}$ ont plus que doublé et celles de $\mathrm{N}_{2} \mathrm{O}$ ont crû de $20 \%$, poussant le climat à se réchauffer. Du fait de la contribution majeure de ces gaz au changement climatique, il est naturel que les scientifiques et les agences spatiales s'intéressent à leur mesure depuis l'espace, particulièrement pour $\mathrm{CO}_{2}$ et $\mathrm{CH}_{4}$ qui bénéficient déjà de missions dédiées (à la différence de $\mathrm{N}_{2} \mathrm{O}$ ). Mais, comme nous le verrons, ces gaz posent des problèmes inédits par rapport aux autres espèces chimiques couramment mesurées depuis l'espace, comme la vapeur d'eau ou l'ozone, et leur télédétection n'a pas encore atteint le niveau de précision nécessaire pour en tirer un bénéfice clair sur la connaissance du cycle du carbone ou de l'azote. Le présent article décrit les enjeux de ce nouveau défi pour l'observation spatiale, en abordant successivement les objectifs de cette quête, le principe de la mesure, les difficultés qu'elle rencontre et les missions satellitaires qui les abordent.

\section{Objectifs}

Les gaz à effet de serre à longue durée de vie s'accumulent dans l'atmosphère tout en étant brassés par la circulation atmosphérique. Ils varient donc relativement peu spatialement. La concentration des colonnes totales de $\mathrm{CO}_{2}$ (concentration moyenne de $\mathrm{CO}_{2}$ entre la surface et le sommet de l'atmosphère), par exemple, varie aujourd'hui de quelques pourcents au maximum autour de $400 \mathrm{ppm}^{1}$ (voir figure 1, en haut). À la surface du globe, les mesures de concentrations précises $(\approx 0,1 \mathrm{ppm})$ ont débuté en 1959 à Mauna Loa dans le Pacifique et sont aujourd'hui acquises sur quelques centaines de sites, comme ceux de la très grande infrastructure de recherche européenne $\mathrm{ICOS}^{2}$. Les variations du $\mathrm{CO}_{2}$ au-delà de ces sites ou sur la verticale sont en général trop faibles pour motiver le fait de les

1. Parties par million: 1 ppm correspond à une molécule sur un million de molécules d'air. 2. Integrated Carbon Observing System, https://icos-atc.lsce.ipsl.fr/ 


\section{Abstract}

\section{Quantifying the sources and sinks of greenhouse gases: a new ambition for space borne remote sensing}

The long lifetime of some greenhouse gases in the atmosphere, such as carbon dioxide, methane and nitrous oxide, gives them a critical role in climate change. At the same time, the accumulation of these gases increases their background level and therefore reduces their space-time variability relative to this level, which leads to particularly challenging specifications on the expected accuracy of such measurements to bring new information. Their remote sensing from space has therefore started late, in the 2000s. The measurement of their atmospheric concentration is essentially motivated by the quantification of the amount of gas exchanged between the atmosphere and the Earth's surface. Such an objective requires the development of sophisticated processing chains, including atmospheric chemistrytransport models and other complementary sources of information on the fluxes of these gases. compléter par de coûteuses missions spatiales, si l'on ne s'intéresse qu'aux concentrations du $\mathrm{CO}_{2}$. La question est similaire pour $\mathrm{CH}_{4}$ et $\mathrm{N}_{2} \mathrm{O}$, même si ces molécules bénéficient de moins de sites de mesure.

En revanche, certaines des sources (émissions) et tous les puits (absorption) de ces gaz à la surface du globe qui, joints au transport et à la chimie atmosphériques, régissent les petites variations des concentrations dans l'atmosphère, sont mal connus. Or, ces sources et puits varient notablement dans l'espace et dans le temps. Cette méconnaissance est amplifiée par la diversité des processus d'émission et d'absorption sous-jacents : dissolution dans l'océan, photosynthèse des plantes, respiration des organismes, combustion d'énergie fossile, etc. pour le $\mathrm{CO}_{2}$; décomposition de la matière organique dans les zones humides ou les décharges, digestion des ruminants, feux de biomasse, exploitation des énergies fossiles, etc. pour le $\mathrm{CH}_{4}$; oxydation de l'azote des sols (stimulée par exemple par l'utilisation d'engrais agricoles), chimie industrielle, décomposition du fumier, production, distribution et combustion d'énergie fossile, etc. pour le $\mathrm{N}_{2} \mathrm{O}$. La connaissance et la compréhension des mécanismes
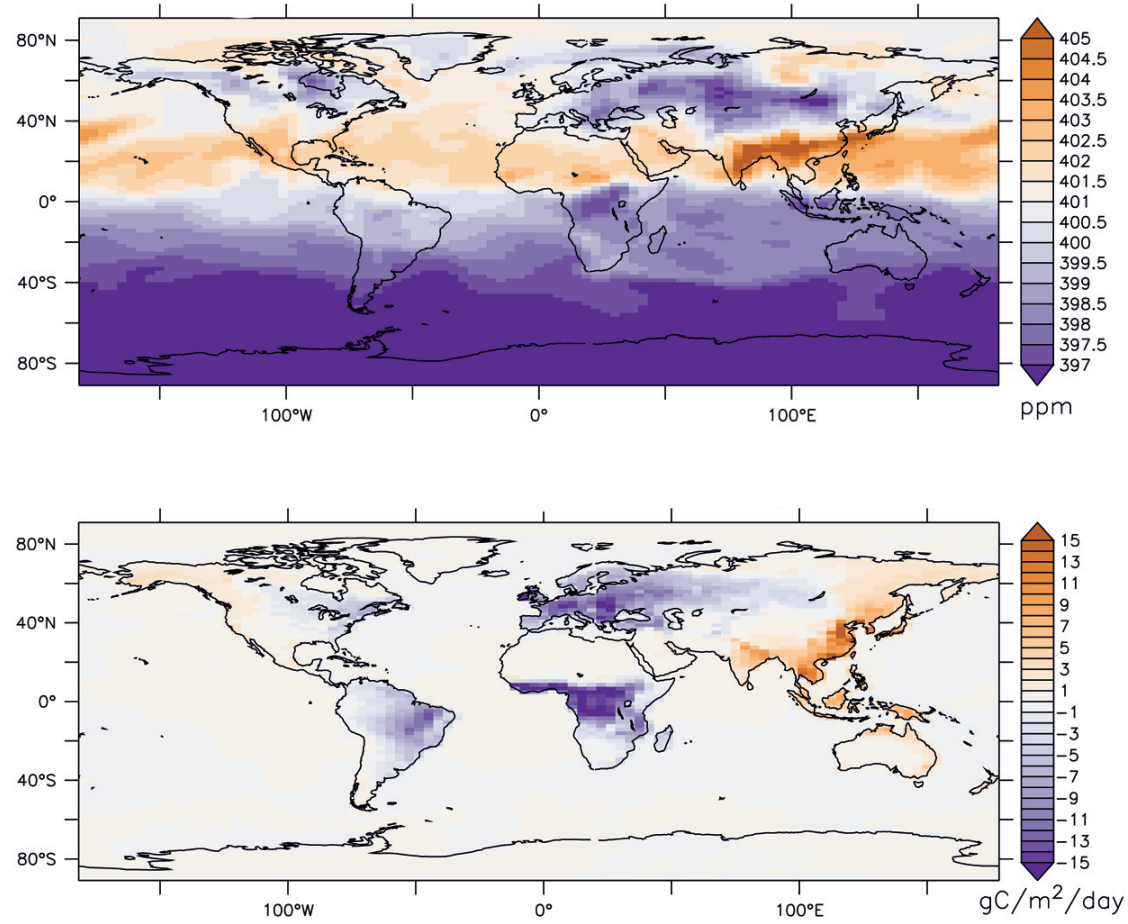

Figure 1. Estimation des flux de surface de $\mathrm{CO}_{2}\left(\mathrm{~g} \mathrm{C} \mathrm{m}^{-2}\right.$ jour-1, en bas) et de la colonne totale (ppm, en haut) au 2 juin 2015 à 12 h 00 TU. Par convention, les valeurs négatives des flux représentent des puits (comme sur la plupart des écosystèmes terrestres tempérés de l'hémisphère Nord, pendant la saison de croissance) et les valeurs positives indiquent des émissions (comme en Inde avant la mousson d'été). Source : service atmosphérique Copernicus (http://atmosphere.copernicus.eu).

d'émission et d'absorption sous-jacents sont pourtant nécessaires pour anticiper l'évolution du climat. La quantification des sources et des puits est aussi à la base des politiques de lutte contre le réchauffement climatique : quantifier les sources pour les réglementer et surveiller les diminutions promises par les États (par exemple dans le cadre de l'Accord de Paris sur le climat), quantifier les puits naturels pour les préserver et les renforcer. L'échelle de temps ciblée pour cette application correspond essentiellement à l'année, résolution temporelle des comptabilités fournies à la Convention cadre des Nations unies sur les changements climatiques par les États, alors que les échelles plus courtes servent plutôt à la compréhension des mécanismes.

La mesure des gaz à effet de serre à longue durée de vie depuis l'espace est donc essentiellement motivée par l'information qu'ils contiennent sur leurs flux de surface (somme des sources et des puits à la surface du globe).

\section{Principe}

Les instruments de mesure embarqués sur des satellites ne rendent pas compte directement de la composition chimique de l'atmosphère. Ils mesurent le rayonnement dans des bandes spectrales où les molécules étudiées sont actives. Connaissant l'intensité de la source du rayonnement et avec l'aide d'un modèle de transfert radiatif qui permet de simuler l'interaction de la matière avec ce rayonnement, on peut en déduire le nombre de molécules de l'espèce étudiée. Trois sources de rayonnement peuvent être utilisées. La première est le soleil : la mesure des luminances (vers 1,6 et $2,0 \mu \mathrm{m}$ pour le $\mathrm{CO}_{2}$, vers 1,7 et 2,3 $\mu \mathrm{m}$ pour $\mathrm{CH}_{4}$ ) renseigne alors sur la colonne totale du gaz lorsque l'instrument vise la surface du globe (on exclut ici et dans la suite les mesures au limbe - visée directe du soleil -, car elles renseignent sur les concentrations dans la haute atmosphère et ne servent pas pour l'estimation des flux de surface). La seconde est le rayonnement thermique de la Terre et de son atmosphère : la mesure (vers 4,3 et $15 \mu \mathrm{m}$ pour $\mathrm{CO}_{2}$, vers 4,5 et $7,8 \mu \mathrm{m}$ pour $\mathrm{CH}_{4}$ et $\mathrm{N}_{2} \mathrm{O}$ ) permet alors de restituer une colonne partielle couvrant essentiellement la moyenne et la haute troposphère. La troisième est le rayonnement laser à des longueurs d'onde bien choisies, qui informe aussi sur la colonne totale. Dans les trois 


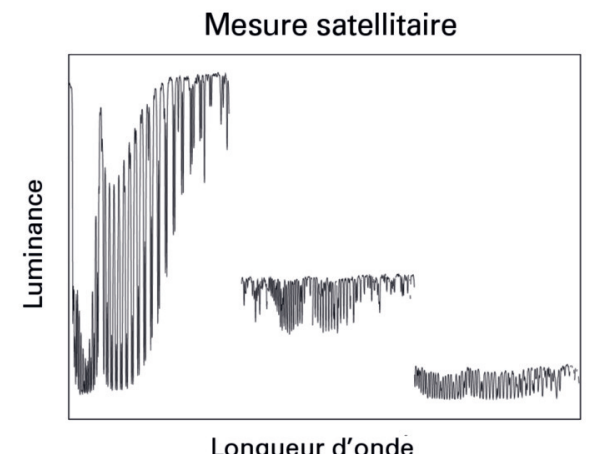

\section{Concentration a priori}

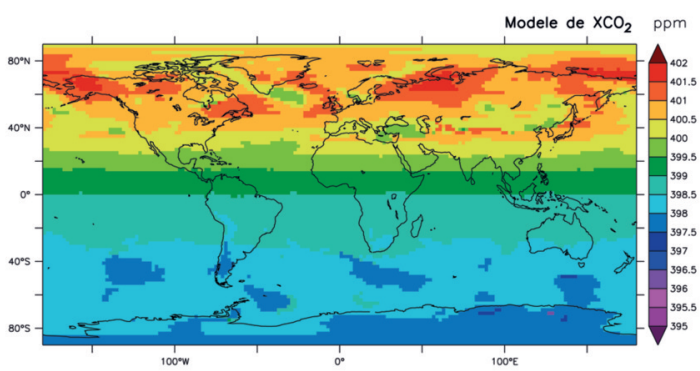

Flux a priori
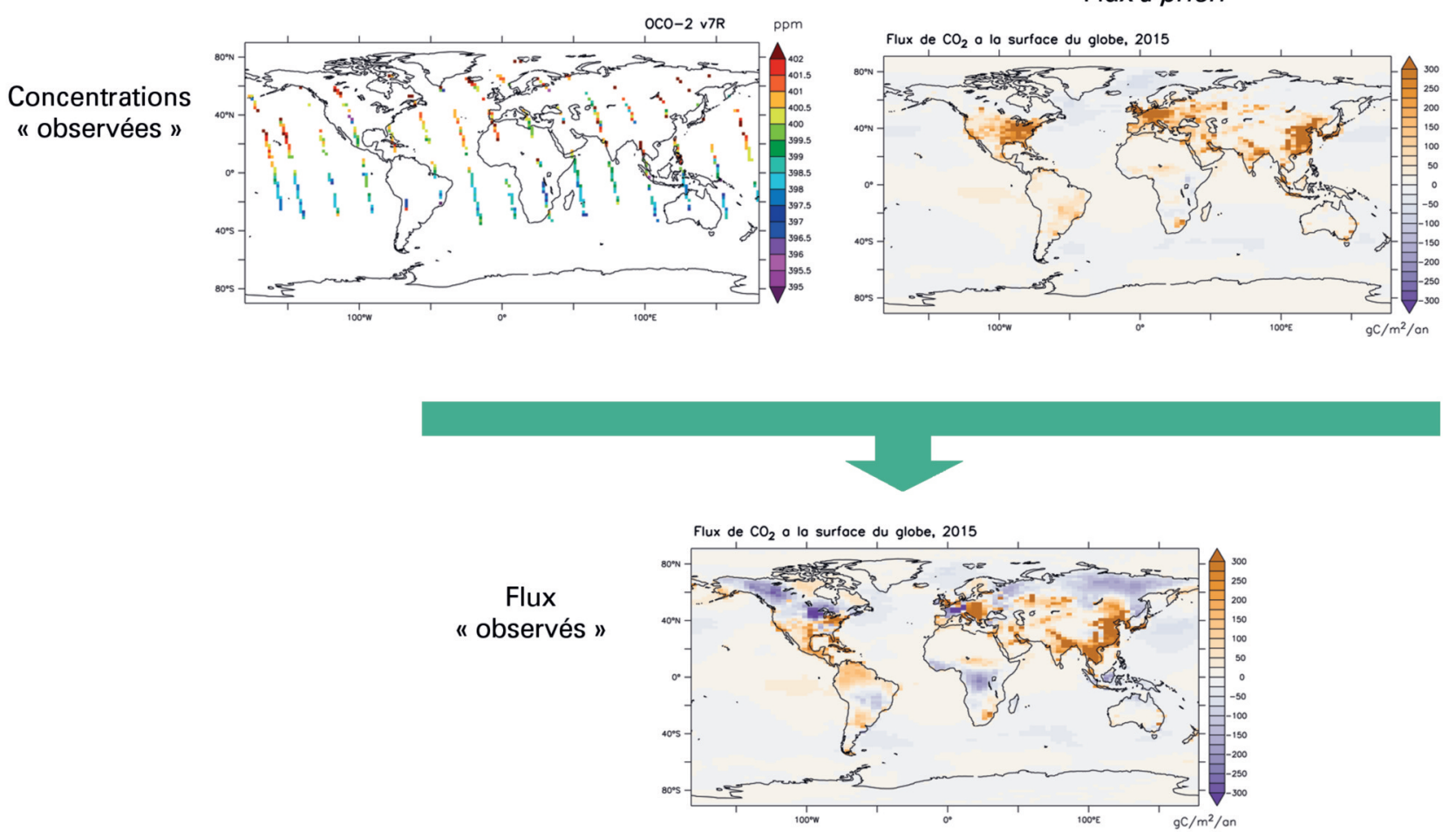

Figure 2. Illustration des étapes nécessaires à l'estimation des flux de surface de $\mathrm{CO}_{2}$ (ligne du bas ; carte annuelle obtenue par le service atmosphérique Copernicus) à partir : (i) de mesures spectrales faites par satellite (ligne du haut, à gauche; une observation de 0CO-2 dans trois bandes spectrales), (ii) d'une information a priori sur les colonnes de $\mathrm{CO}_{2}$ (ligne du haut, à droite ; carte utilisée pour l'inversion des mesures spectrales OCO-2) et (iii) d'une information a priori sur les flux de $\mathrm{CO}_{2}$ (ligne du milieu, à droite ; carte annuelle utilisée par le service atmosphérique Copernicus). L'estimation des concentrations de $\mathrm{CO}_{2}$ est une étape intermédiaire (ligne du milieu, à gauche; mesures de la colonne de $\mathrm{CO}_{2}$ obtenues avec OCO-2). Sources : service atmosphérique Copernicus (http://atmosphere.copernicus.eu/) et portail OCO-2 de JPL-Caltech (http://co2.jpl.nasa.gov).

cas, le problème d'estimation de la concentration à partir des luminances mesurées est un problème inverse qui est mathématiquement « mal posé » (c'est-à-dire qu'une infinité de solutions est possible), ne serait-ce que parce que les mesures radiométriques sont bruitées : il est nécessaire de le régulariser en apportant une information complémentaire, dite a priori, provenant de climatologies ou de modèles. Ce processus est illustré dans les lignes du haut et du milieu de la figure 2. La non-unicité de la solution est renforcée par la contribution d'éléments autres que les gaz ciblés dans le rayonnement mesuré, sur lesquels nous ne disposons pas toujours d'éléments fiables, ni dans les spectres ni par d'autres mesures ou par des modèles : eau liquide ou solide des nuages, aérosols, albédo de la surface, etc. On notera que la télédétection par laser est moins sensible aux aérosols et aux nuages que la télédétection passive, mais plus difficile à mettre en œuvre.

Comme nous l'avons vu, l'estimation des concentrations de gaz à effet de serre ne motive pas directement ces mesures : leur aboutissement réside dans l'extraction de l'information sur les flux des gaz en question (cf. figure 1 pour le $\left.\mathrm{CO}_{2}\right)$. Le principe de cette extraction est également un problème inverse, comme pour l'estimation des concentrations, mais le modèle physique est alors un modèle de chimie-transport atmosphérique. Dans cette deuxième étape (illustrée dans les lignes du milieu et du bas de la figure 2), le problème est aussi mal posé : une infinité de distributions spatiotemporelles des flux pourrait mener aux concentrations observées, d'abord parce que les mesures ne sont pas disponibles partout, ensuite parce qu'elles sont bruitées, enfin parce que le brassage atmosphérique provoque la perte irrémédiable d'une partie de l'information sur les flux. L'information 
a priori sur les flux est aussi souvent fournie par des climatologies et par des sorties de modèles, mais d'autres types d'estimations (à partir de données socio-économiques ou d'observations des surfaces brûlées, par exemple) sont aussi utilisés. Ce manque de "pureté » des produits finaux vis-à-vis de la mesure satellitaire a souvent été mitigé par un argument sur une sensibilité relativement faible de l'étape d'estimation des flux utilisant des mesures de colonne totale (Rayner et O’Brien, 2001 ; Olsen et Randerson, 2004 ; Yang et al., 2007) spatialement denses (Kang et al., 2012) à la qualité du modèle de transport utilisé. Nous verrons qu'il n'en est rien.

\section{Défis}

Les concentrations de $\mathrm{CO}_{2}, \mathrm{CH}_{4}$ et $\mathrm{N}_{2} \mathrm{O}$ dans l'atmosphère sont croissantes mais restent faibles (autour de 400, 1,85 et $0,33 \mathrm{ppm}$, respectivement), ce qui nécessite des capacités de détection très précises. Par ailleurs, nous avons indiqué plus haut qu'elles varient peu dans l'espace et dans le temps, alors que ce sont précisément leurs faibles variations qu'il s'agit de mesurer. Ainsi, les simulations numériques des colonnes totales de $\mathrm{CO}_{2}$ et $\mathrm{CH}_{4}$ faites par le service atmosphérique Copernicus ${ }^{3}$ de la Commission européenne à la résolution mondiale de $15 \mathrm{~km}$ indiquent une variabilité sur le globe et sur 1 an caractérisée par un écart type de seulement $3,5 \mathrm{ppm}$ pour le $\mathrm{CO}_{2}(0,9 \%$ de la valeur moyenne) et de $0,08 \mathrm{ppm}$ pour le $\mathrm{CH}_{4}$ (4\% de la valeur moyenne), cette variabilité comprenant les accroissements dus aux activités humaines. De telles simulations sont quasiment indépendantes des mesures de $\mathrm{CO}_{2}$ et de $\mathrm{CH}_{4}$ et reproduisent pourtant les mesures de la colonne de ces gaz avec une précision plus élevée que leur variabilité (Massart et al., 2014 ; Agustí-Panareda et al., 2016). L'information supplémentaire que la télédétection spatiale peut apporter est donc ténue. En d'autres termes, les flux de surface à proximité de la mesure n'expliquent en général qu'une toute petite partie des concentrations mesurées. Par conséquent, les spécifications sur les mesures de colonnes sont remarquablement contraignantes : d'après ESA (2014), les biais sur les colonnes totales doivent être inférieurs à $0,5 \mathrm{ppm}$ pour le $\mathrm{CO}_{2}$

3. http://atmosphere.copernicus.eu
$(0,12 \%$ de la valeur moyenne $)$ et $10 \mathrm{ppb}$ pour le $\mathrm{CH}_{4}(0,54 \%$ de la valeur moyenne), alors même que l'interprétation des spectres mesurés est pénalisée par un ensemble d'incertitudes sur les modèles spectroscopiques et sur les autres variables atmosphériques (concentrations d'autres espèces gazeuses ou d'aérosols, etc.) ou de la surface (albédo) qui influent sur le spectre. Concernant les mesures des concentrations dans la troposphère libre obtenues avec les mesures du rayonnement thermique, les spécifications sont encore plus serrées, puisque la variabilité des concentrations diminue avec l'altitude. En revanche, dans le cas d'émissions intenses (par exemple, des feux ou de grosses centrales électriques fonctionnant avec des combustibles fossiles), les spécifications pourraient être relâchées, puisque le signal est alors plus fort. Notons cependant que les émissions anthropiques sont mieux connues que les flux naturels; apporter des connaissances nouvelles conduit à des exigences plus élevées.

Les spécifications drastiques sur les produits de colonnes s'appliquent aussi aux modèles de chimie-transport qui simulent ces colonnes pour estimer ensuite les flux. Il est difficile de quantifier leur erreur, mais les exercices numériques d'intercomparaison suggèrent une forte sensibilité des flux retrouvés aux caractéristiques des modèles de transport sous-jacents (Chevallier et al., 2010 ; Houweling et al., 2010), contrairement au postulat initial (Rayner et O’ Brien, 2001). La sophistication inégale des modèles participant à ces exercices aggrave probablement le diagnostic, mais on devine déjà l'importance de la qualité du modèle de transport dans le cas de $\mathrm{CO}_{2}$ sur la figure 1 : la variabilité de la colonne apparaît très différente de celle des flux de surface, à la suite du transport atmosphérique.

La validation des flux estimés pose aussi des difficultés. En effet, les mesures de référence pour les flux sont celles faites ponctuellement par la méthode des corrélations turbulentes (Aubinet et al., 2012). Leur résolution varie entre quelques hectares et quelques kilomètres carrés seulement, suivant les conditions. À moins peut-être de disposer d'une capacité d'imagerie, les flux de surface sont estimés à partir des mesures spatiales à bien plus basse résolution. Il n'existe donc pas de mesures pour valider directement les flux des gaz à effet de serre estimés à l'aide des satellites, alors qu'ils sont l'objectif de ces missions.

Pour les colonnes totales, la validation s'effectue par comparaison avec des mesures de télédétection depuis le sol étalonnées avec des mesures conventionnelles (par prélèvement d'air) par avion (Wunch et al., 2011). À ce jour, elle montre en général des différences systématiques de l'ordre du ppm pour le $\mathrm{CO}_{2}$ et de quelques ppb pour le $\mathrm{CH}_{4}$. Certains des biais constatés dépendent de variables qui sont elles-mêmes corrélées avec les flux de surface, pénalisant l'analyse des signaux. C'est le cas de celle de l'albédo de surface, qui peut être lié à un changement de type de source ou de puits (par exemple, un albédo faible sur les forêts, puits de $\mathrm{CO}_{2}$, et fort sur les zones urbanisées, émettrices de $\mathrm{CO}_{2}$ ). La dépendance des biais suivant les caractéristiques de la scène étudiée fausse aussi le suivi des panaches d'émission au cours de leur dispersion.

Les mesures satellitaires fournissent une densité de mesures à grande échelle inaccessible par d'autres moyens, mais n'offrent pas forcément pour autant de couverture mondiale, ni même de couverture complète dans leur zone d'observation, fut-elle hémisphérique depuis une orbite géostationnaire. Par exemple, les mesures de colonnes à partir du rayonnement solaire réfléchi sont aveugles dans les hautes latitudes de l'hémisphère d'hiver. Elles ne sont pas non plus opérantes en présence de nuages ou d'aérosols épais. Ces lacunes impliquent que le cycle saisonnier des flux peut être inégalement contraint par les mesures.

\section{Satellites}

L'ensemble des défis décrits ci-dessus est relevé par un nombre croissant de missions satellitaires, car les agences spatiales ont pris la mesure de l'importance stratégique de l'objectif final.

Les premiers produits satellitaires sur les concentrations de $\mathrm{CO}_{2}$ ont été générés à partir des mesures dans l'infrarouge thermique des instruments Tovs embarqués sur les satellites opérationnels en orbite terrestre basse (polaire) de la Noaa (Chédin et al., 2003). Ils ont permis de restituer une information dans les tropiques 
cohérente avec des mesures aéroportées loin des principaux sources et puits, même si les instruments n'avaient pas été conçus pour cet objectif. Des instruments à plus haute résolution spectrale (principalement Iasi à bord des satellites opérationnels Metop ESA/Eumetsat -, mais aussi Airs et TES sur Aqua et Aura - Nasa) continuent les séries temporelles de ce type de mesure, toujours en orbite basse. Des mesures équivalentes sont aussi produites pour le $\mathrm{CH}_{4}$ (Xiong et al., 2008 ; Crevoisier et al., 2009) et, plus difficilement, pour $\mathrm{N}_{2} \mathrm{O}$ (Ricaud et al., 2009). Ces produits sont peu utilisées pour estimer les flux de surface (Chevallier et al., 2005 ; Nassar et al., 2011 et Cressot et al., 2014, sont trois exceptions avec des succès inégaux) parce que certains sont trop biaisés et parce qu'ils ne couvrent pas la basse troposphère où les concentrations mesurées peuvent être reliées plus directement aux flux de surface. L'instrument Sciamachy à bord d'Envisat (ESA), opérationnel de 2002 à 2012, a permis les premières mesures satellitaires des colonnes totales de $\mathrm{CO}_{2}$ et $\mathrm{CH}_{4}$. Malgré les polémiques induites par une communication initiale parfois hasardeuse (Frankenberg et al., 2005, 2008 ; Buchwitz et al., 2005), elles ont ouvert la voie au traitement des missions dédiées qui ont suivi : le satellite japonais Gosat ${ }^{4}$ lancé en 2009 pour observer $\mathrm{CO}_{2}$ et $\mathrm{CH}_{4}$, le satellite américain $\mathrm{OCO}-2$ (Nasa) dédié au $\mathrm{CO}_{2}$ et lancé en 2014, le satellite chinois Tansat ${ }^{5}$ dédié aussi au $\mathrm{CO}_{2}$ et lancé en 2016.

En termes d'estimation des flux de surface, les résultats obtenus pour le méthane jusqu'à présent montrent l'augmentation de la qualité des mesures entre Sciamachy et Gosat (Monteil et al., 2013 ; Alexe et al., 2015) et convergent vers une réévaluation à la hausse des émissions des zones humides dans les régions tropicales (Monteil et al., 2013). Elles ont permis des études d'émissions régionales (Wecht et al., 2014), incluant pour certaines les sources ponctuelles intenses (Kort et al., 2014 ; Schneising et al., 2014). Dans ces résultats, l'impact des incertitudes sur le transport atmosphérique reste sensible (Locatelli et al., 2015), mais la situation est bien plus critique pour le $\mathrm{CO}_{2}$, car les résultats régionaux des différents groupes divergent fortement en fonction du modèle de transport utilisé et des produits satellitaires assimilés (Chevallier et al., 2014, 2016; Houweling et al., 2015). Dans certaines configurations, les résultats dans quelques régions du globe peuvent sembler réalistes (Guerlet et al., 2013 ; Basu et al., 2014 ; Reuter et al., 2014 ; Detmers et al., 2015), mais l'ensemble manque encore de cohérence.

Plusieurs missions sont attendues dans un avenir proche, toujours en orbite basse : Sentinel-5P (ESA, prévu pour 2017) permettra de mesurer les colonnes totales de $\mathrm{CH}_{4}$ parmi d'autres espèces et l'instrument américain OCO-3 (Nasa, prévu pour 2018 à bord de la Station spatiale internationale) sera dédié au $\mathrm{CO}_{2}$. Ces missions imminentes offriront une meilleure densité de mesures. D'autres devraient aussi fournir des mesures de plus haute qualité. Gosat-2 prendra la relève de Gosat vers 2018. En France, le Cnes prépare les satellites Merlin (prévu pour 2021) en partenariat avec le $\mathrm{DLR}^{6}$ en Allemagne, qui inclura pour la première fois une technologie active (laser) pour observer le $\mathrm{CH}_{4}$, et Microcarb (prévu vers 2020) pour le $\mathrm{CO}_{2}$, avec une nouvelle bande spectrale qui devrait fournir des mesures moins sensibles à la présence des diffuseurs atmosphériques. Après plusieurs études préliminaires ${ }^{7}, 1^{\prime}$ Europe continue à préparer une mission pour observer le $\mathrm{CO}_{2}$ avec un accent important mis sur les émissions intenses à fine échelle ${ }^{8}$. Enfin, d'autres concepts innovants sont à l'étude : la télédétection par laser du $\mathrm{CO}_{2}$ (projet de satellite Ascends de la Nasa) et la mesure de $\mathrm{CO}_{2}$ et de $\mathrm{CH}_{4}$ depuis une orbite géostationnaire (projet de satellite GeoCarb de la Nasa). Il n'y a pour l'instant pas de mission à l'étude pour le $\mathrm{N}_{2} \mathrm{O}$, pénalisé par ses faibles concentrations et gradients dans l'atmosphère, mais le futur instrument Iasi-NG sur Metop-SG (ESA/Eumetsat) devrait permettre de progresser à partir de 2021.

\section{Conclusion}

La télédétection spatiale des gaz à effet de serre à longue durée de vie tente de répondre à une demande croissante d'information sur les principaux moteurs du changement climatique. Elle a pour ambition affichée de contribuer à la compréhension des cycles biogéochimiques d'une part, et au suivi des politiques de réglementation des flux de ces gaz, d'autre part. Les résultats actuels pour le $\mathrm{CH}_{4}$ s'en rapprochent progressivement, mais restent très en deçà pour le $\mathrm{CO}_{2}$ et sont encore embryonnaires pour le $\mathrm{N}_{2} \mathrm{O}$. Les chaînes de traitement, développées au sein d'une forte coopération internationale et d'une communauté scientifique croissante, rassemblent des savoirs très variés, de la physique du rayonnement à la physique du transport et de la chimie dans l'atmosphère, qui tous doivent être encore affinés. Des progrès technologiques sont aussi attendus, par exemple dans le secteur stratégique des détecteurs pour les rendre plus sensibles. L'ensemble des défis rencontrés stimule et renouvelle les problématiques liées à la télédétection de l'atmosphère depuis l'espace.

4. Japan Aerospace Exploration Agency/National Institute for Environmental Studies/Ministry of the Environment

5. Chinese Academy of Sciences/Ministry of Science and Technology

6. Deutsches Zentrum für Luft- und Raumfahrt

7. ESA $(2008,2015)$

8. Commission européenne (2015)

\section{Bibliographie}

Agustí-Panareda A., Massart S., Chevallier F., Balsamo G., Boussetta S., Dutra E., Beljaars A., 2016. A biogenic $\mathrm{CO}_{2}$ flux adjustment scheme for the mitigation of large-scale biases in global atmospheric $\mathrm{CO}_{2}$ analyses and forecasts. Atmos. Chem. Phys., 16, 10399-10418. doi: 10.5194/acp-2015-987

Alexe M., Bergamaschi P., Segers A., Detmers R., Butz A., Hasekamp O., Guerlet S., Parker R., Boesch H., Frankenberg C., Scheepmaker R.A., Dlugokencky E., Sweeney C., Wofsy S.C., Kort E.A., 2015. Inverse modelling of $\mathrm{CH}_{4}$ emissions for 2010-2011 using different satellite retrieval products from GOSAT and SCIAMACHY. Atmos. Chem. Phys., 15, 113-133. doi: 10.5194/acp-15-113-2015

Aubinet M., Vesala T., Papale D., 2012. Eddy Covariance: A Practical Guide to Measurement and Data Analysis. Springer, $460 \mathrm{p}$.

Basu S., Krol M., Butz A., Clerbaux C., Sawa Y., Machida T., Matsueda H., Frankenberg C., Hasekamp O.P., Aben I., 2014. The seasonal variation of the CO 2 flux over Tropical Asia estimated from GOSAT, CONTRAIL, and IASI. Geophys. Res. Lett., 41, 1809-1815. doi: 10.1002/2013GL059105

Buchwitz M., de Beek R., Burrows J.P., Bovensmann H., Warneke T., Notholt J., Meirink J.F., Goede A.P.H., Bergamaschi P., Körner S., Heimann M., Schulz A., 2005. Atmospheric methane and carbon dioxide from SCIAMACHY satellite data: initial comparison with chemistry and transport models, Atmos. Chem. Phys., 5, 941-962. doi: 10.5194/acp-5-941-2005 
Chédin A., Serrar S., Scott N.A., Crevoisier C., Armante R., 2003. First global measurement of midtropospheric $\mathrm{CO}_{2}$ from NOAA polar satellites: Tropical zone. J. Geophys. Res., 108, 4581. doi: 10.1029/2003JD003439

Chevallier F., Fisher M., Peylin P., Serrar S., Bousquet P., Bréon F.-M., Chédin A., Ciais P., 2005. Inferring $\mathrm{CO}_{2}$ sources and sinks from satellite observations: method and application to TOVS data. J. Geophys. Res., 110, D24309. doi: 10.1029/2005JD006390

Chevallier F., Feng L., Boesch H., Palmer P., Rayner P., 2010. On the impact of transport model errors for the estimation of $\mathrm{CO}_{2}$ surface fluxes from GOSAT observations. Geophys. Res. Lett., 37, L21803. doi: 10.1029/2010GL044652

Chevallier F., Palmer P.I., Feng L., Boesch H., O'Dell C.W., Bousquet P., 2014. Towards robust and consistent regional $\mathrm{CO}_{2}$ flux estimates from in situ and space-borne measurements of atmospheric $\mathrm{CO}_{2}$. Geophys. Res. Lett., 41, 1065-1070. doi: 10.1002/2013GL058772

Chevallier F., Alexe M., Bergamaschi P., Brunner D., Feng L., Houweling S., Kaminski T., Knorr W., van Leeuwen T.T., Marshall J., Palmer P.., Scholze M., Sundström A.-M., Voßbeck M., 2016. Climate Assessment Report for the GHG-CCl project of ESA's Climate Change Initiative, 94 p., version 3, 3 May 2016.

Commission européenne, 2015. Towards a European Operational Observing System to Monitor Fossil $\mathrm{CO}_{2}$ emissions, Final Report from the expert group, 65 p., disponible à l'adresse : www.copernicus.eu/sites/default/files/library/ $/ \mathrm{CO}_{2}$ Report_220ct2015.pdf

Cressot C., Chevallier F., Bousquet P., Crevoisier C., Dlugokencky E.J., Fortems-Cheiney A., Frankenberg C., Parker R., Pison I., Scheepmaker R.A., Montzka S.A., Krummel P.B., Steele L.P., Langenfelds R.L., 2014. On the consistency between global and regional methane emissions inferred from SCIAMACHY, TANSO-FTS, IASI and surface measurements. Atmos. Chem. Phys., 14, 577-592. doi: 10.5194/acp-14-577-2014

Crevoisier C., Nobileau D., Fiore A.M., Armante R., Chédin A., Scott N.A., 2009. Tropospheric methane in the tropics - first year from IASI hyperspectral infrared observations. Atmos. Chem. Phys., 9, 6337-6350. doi: 10.5194/acp-9-6337-2009

Detmers R.G., Hasekamp 0., Aben I., Houweling S., van Leeuwen T.T., Butz A., Landgraf J., Köhler P., Guanter L., Poulter B., 2015. Anomalous carbon uptake in Australia as seen by GOSAT. Geophys. Res. Lett., 42, 8177-8184. doi: 10.1002/2015GL065161

ESA, 2008. A-SCOPE, Report for Assessment, SP-1313/1, 109 p., disponible à l'adresse : http://esamultimedia.esa.int/docs/SP1313-1_ASCOPE.pdf

ESA, 2014. User Requirements Document for the GHG-CCI project of ESA's Climate Change Initiative, p. 38, version 2, 28 Aug. 2014, disponible à l'adresse : www.esa-ghg-cci.org/?q=webfm_send/173

ESA, 2015. CARBONSAT, Report for Mission Selection, SP-1330/1, 207 p., disponible à l'adresse : http://esamultimedia.esa.int/docs/Earth0bservation/SP1330-1_CarbonSat.pdf

Frankenberg C., Meirink J.F., van Weele M., Platt U., Wagner T., 2005. Assessing methane emissions from global space-borne observations. Science, 308, 1010-1014. doi: 10.1126/science.1106644

Frankenberg C., Bergamaschi P., Butz A., Houweling S., Meirink J.F., Petersen K., Schrijver H., Warneke T., Aben I., 2008. Tropical methane emissions: A revised view from SCIAMACHY onboard Envisat. Geophys. Res. Lett., 35, L15811. doi: 10.1029/ 2008GL034300

Guerlet S., Basu S., Butz A., Krol M., Hahne P., Houweling S., Hasekamp O.P., Aben I., 2013. Reduced carbon uptake during the 2010 Northern Hemisphere summer from GOSAT. Geophys. Res. Lett., 40, 2378-2383. doi: 10.1002/grl.50402

Houweling S., Aben I., Bréon F.-M., Chevallier F., Deutscher N., Engelen R., Gerbig C., Griffith D., Hungershoefer K., Macatangay R., Marshall J., Notholt J., Peters W., Serrar S., 2010. The importance of transport model uncertainties for the estimation of $\mathrm{CO}_{2}$ sources and sinks using satellite measurements. Atmos. Chem. Phys., 10, 9981-9992. doi: 10.5194/acp-10-9981-2010

Houweling S., Baker D., Basu S., Boesch H., Butz A., Chevallier F., Deng F., Dlugokencky E.J., Feng L., Ganshin A., Hasekamp O., Jones D., Maksyutov S., Marshall J., Oda T., Dell C.W.O., Oshchepkov S., Palmer P.I., Peylin P., Poussi Z., Reum F., Takagi H., Yoshida Y., Zhuravlev R., 2015. An intercomparison of inverse models for estimating sources and sinks of $\mathrm{CO}_{2}$ using GOSAT measurements. J. Geophys. Res. Atmos., 120, 5253-5266. doi: 10.1002/2014JD022962

Kang J.-S., Kalnay E., Miyoshi T., Liu J., Fung I., 2012. Estimation of surface carbon fluxes with an advanced data assimilation methodology. J. Geophys. Res., 117, D24101. doi: 10.1029/2012JD018259

Kort E.A., Frankenberg C., Costigan K.R., Lindenmaier R., Dubey M.K., Wunch D., 2014. Four corners: The largest US methane anomaly viewed from space, Geophys. Res. Lett., 41, 6898-6903. doi: 10.1002/2014GL061503

Locatelli R., Bousquet P., Saunois M., Chevallier F., Cressot C., 2015. Sensitivity of the recent methane budget to LMDz sub-grid-scale physical parameterizations. Atmos. Chem. Phys., 15, 9765-9780. doi: 10.5194/acp-15-9765-2015, 2015

Massart S., Agustí-Panareda A., Aben I., Butz A., Chevallier F., Crevoisier C., Engelen R., Frankenberg C., Hasekamp 0., 2014. Assimilation of atmospheric methane products in the MACC-II system: from SCIAMACHY to TANSO and IASI. Atmos. Chem. Phys., 14, 6139-6158. doi: 10.5194/acp-14-6139-2014

Monteil G., Houweling S., Butz A., Guerlet S., Schepers D., Hasekamp 0., Frankenberg C., Scheepmaker R., Aben I., Röckmann T., 2013. Comparison of $\mathrm{CH}_{4}$ inversions based on 15 months of GOSAT and SCIAMACHY observations. J. Geophys. Res. Atmos., 118, 11807-11823. doi: 10.1002/2013JD019760

Nassar R., Jones D.B.A., Kulawik S.S., Worden J.R., Bowman K.W., Andres R.J., Suntharalingam P., Chen J.M., Brenninkmeijer C.A.M., Schuck T.J., Conway T.J., Worthy D.E., 2011. Inverse modeling of $\mathrm{CO}_{2}$ sources and sinks using satellite observations of $\mathrm{CO}_{2}$ from TES and surface flask measurements, Atmos. Chem. Phys., 11, 6029-6047. doi: 10.5194/acp-11-6029-2011

Olsen S.C., Randerson J.T., 2004. Differences between surface and column atmospheric $\mathrm{CO}_{2}$ and implications for carbon cycle research. J. Geophys. Res., 109, D02301. doi: 10.1029/2003JD003968

Rayner P.J., O'Brien D.M., 2001. The utility of remotely sensed $\mathrm{CO}_{2}$ concentration data in surface source inversions. Geophys. Res. Lett., 28, 175-178.

Reuter M., Buchwitz M., Hilker M., Heymann J., Schneising 0., Pillai D., Bovensmann H., Burrows J.P., Bösch H., Parker R., Butz A., Hasekamp 0., O'Dell C.W., Yoshida Y., Gerbig C., Nehrkorn T., Deutscher N.M., Warneke T., Notholt J., Hase F., Kivi R., Sussmann R., Machida T., Matsueda H., Sawa Y., 2014. Satellite-inferred European carbon sink larger than expected. Atmos. Chem. Phys., 14, 13739-13753. doi: 10.5194/acp-14-13739-2014

Ricaud P., Attié J.-L., Teyssèdre H., El Amraoui L., Peuch V.-H., Matricardi M., Schluessel P., 2009. Equatorial total column of nitrous oxide as measured by IASI on MetOp-A: implications for transport processes. Atmos. Chem. Phys., 9, 3947-3956. doi: 10.5194/acp-9-3947-2009

Schneising 0., Burrows J.P., Dickerson R.R., Buchwitz M., Reuter M., Bovensmann H., 2014. Remote sensing of fugitive methane emissions from oil and gas production in North American tight geologic formations. Earth's Future, 2, 548-558. doi: 10.1002/2014EF000265

Wecht K.J., Jacob D.J., Frankenberg C., Jiang Z., Blake D.R., 2014. Mapping of North American methane emissions with high spatial resolution by inversion of SCIAMACHY satellite data. J. Geophys. Res. Atmos., 119, 7741-7756. doi: 10.1002/2014JD021551

Wunch D., Toon G.C., Blavier J.F.L., Washenfelder R.A., Notholt J., Connor B.J., Griffith D.W.T., Sherlock V., Wennberg P.O., 2011. The Total Carbon Column Observing Network. Phil. Trans. R. Soc. A, 369, 2087-2112. doi: 10.1098/rsta.2010.0240

Xiong X., Barnet C., Maddy E., Sweeney C., Liu X., Zhou L., Goldberg M., 2008. Characterization and validation of methane products from the Atmospheric Infrared Sounder (AIRS). J. Geophys. Res., 113, G00A01. doi: 10.1029/2007JG000500

Yang Z., Washenfelder R.A., Keppel-Aleks G., Krakauer N.Y., Randerson J.T., Tans P.P., Sweeney C., Wennberg P.O., 2007. New constraints on Northern Hemisphere growing season net flux. Geophys. Res. Lett., 34, L12807. doi: 10.1029/2007GL029742 